\title{
Stability and boundedness of solutions of a kind of third-order delay differential equations*
}

\author{
A.U. AFUWAPE ${ }^{1}$ and M.O. OMEIKE ${ }^{2}$ \\ ${ }^{1}$ Departmento de Matemáticas, Universidad de Antioquia \\ Calle 67, No. 53-108, Medellín AA 1226, Colombia \\ ${ }^{2}$ Department of Mathematics, University of Agriculture, Abeokuta, Nigeria \\ E-mails: aafuwape@yahoo.co.uk/moomeike@yahoo.com
}

\begin{abstract}
This paper studies the stability and boundedness of solutions of certain nonlinear third-order delay differential equations. Sufficient conditions for the stability and boundedness of solutions for the equations considered are obtained by constructing a Lyapunov functional.
\end{abstract}

\section{Mathematical subject classification: $34 \mathrm{~K} 20$.}

Key words: stability, boundedness, Lyapunov functional, differential equations of third-order with delay.

\section{Introduction}

This paper deals with the stability and boundedness of solution of the delay differential equation

$$
\begin{gathered}
x^{\prime \prime \prime}(t)+h\left(x^{\prime}(t)\right) x^{\prime \prime}(t)+g\left(x^{\prime}(t-r(t))\right)+f(x(t-r(t))) \\
=p\left(t, x(t), x^{\prime}(t), x(t-r(t)), x^{\prime}(t-r(t)), x^{\prime \prime}(t)\right)
\end{gathered}
$$

\#CAM-34/08. Received: 05/XII/08. Accepted: 03/I/10.

*This research was supported by University of Antioquia Research Grant CODI through SUI No. IN10095CE. 
or its equivalent system

$$
\begin{aligned}
x^{\prime} & =y, \\
y^{\prime}= & z, \\
z^{\prime}= & -h(y) z-g(y)-f(x)+\int_{t-r(t)}^{t} g^{\prime}(y(s)) z(s) d s \\
& +\int_{t-r(t)}^{t} f^{\prime}(x(s)) y(s) d s+p(t, x, y, x(t-r(t)), y(t-r(t)), z),
\end{aligned}
$$

where $0 \leq r(t) \leq \gamma, r^{\prime}(t) \leq \beta, 0<\beta<1, \beta$ and $\gamma$ are some positive constants, $\gamma$ will be determined later, $f(x), g(y), h(y), p(t, x, y, x(t-r(t))$, $y(t-r(t)), z)$ are continuous in their respective arguments. Besides, it is supposed that the derivatives $f^{\prime}(x), g^{\prime}(y)$ are continuous for all $x, y$ with $f(0)=$ $g(0)=0$. In addition, it is also assumed that the functions $f(x(t-r(t)))$, $g(y(t-r(t)))$ and $p(t, x, y, x(t-r(t)), y(t-r(t)), z)$ satisfy a Lipschitz condition in $x, y, x(t-r(t)), y(t-r(t))$ and $z$; throughout the paper $x(t), y(t)$ and $z(t)$ are, respectively, abbreviated as $x, y$ and $z$. Then the solution is unique. (See [5, pp. 14]).

In recent year, many books and papers dealt with the delay differential equation and obtained many good results, for example, $[1,2,3,18,19,21]$, etc. In many references, the authors dealt with the problems by considering Lyapunov functions or functionals and obtained the criteria for the stability and boundedness. (See [1-21]).

In particular, recently, Tunç [15], obtained sufficient conditions which ensure the stability and the boundedness of systems

$$
x^{\prime \prime \prime}+a_{1} x^{\prime \prime}+f_{2}\left(x^{\prime}(t-r(t))\right)+a_{3} x=0
$$

and

$x^{\prime \prime \prime}+a_{1} x^{\prime \prime}+f_{2}\left(x^{\prime}(t-r(t))\right)+a_{3} x=p\left(t, x, x^{\prime}, x(t-r(t)), x^{\prime}(t-r(t)), x^{\prime \prime}\right)$,

where $r(t)$ is as defined above, $a_{1}$ and $a_{3}$ are some positive constants.

Our objective in this paper is to establish some sufficient conditions for the stability and for the boundedness of solutions of (1.1) in the cases $p \equiv 0$, $p \neq 0$, respectively. 


\section{Stability}

First, we will give the stability criteria for the general autonomous delay differential system. We consider

$$
x^{\prime}=f\left(x_{t}\right), \quad x_{t}=x(t+\theta), \quad-r \leq \theta \leq 0, t \geq 0,
$$

where $f: C_{H} \longrightarrow \mathbb{R}^{n}$ is a continuous mapping, $f(0)=0, C_{H}:=\{\phi \in$ $\left.\left(C[-r, 0], \mathbb{R}^{n}\right):\|\phi\| \leq H\right\}$ and for $H_{1}<H$, there exists $L\left(H_{1}\right)>0$, with $|f(\phi)| \leq L\left(H_{1}\right)$ when $\|\phi\| \leq H_{1}$.

Definition 2.1. An element $\psi \in C$ is in the $\omega$-limit set of $\phi$, say $\Omega(\phi)$, if $x(t, 0, \phi)$ is defined on $[0, \infty)$ and there is a sequence $\left\{t_{n}\right\}, t_{n} \longrightarrow \infty$, as $n \longrightarrow$ $\infty$, with $\left\|x_{t_{n}}(\phi)-\psi\right\| \longrightarrow 0$ as $n \longrightarrow \infty$ where $x_{t_{n}}(\phi)=x\left(t_{n}+\theta, 0, \phi\right)$ for $-r \leq \theta \leq 0$.

Definition 2.2 (See [17]). A set $Q \subset C_{H}$ is an invariant set if for any $\phi \in Q$, the solution of $(2.1), x(t, 0, \phi)$, is defined on $[0, \infty)$, and $x_{t}(\phi) \in Q$ for $t \in[0, \infty)$.

Lemma 2.1 (See [13]). If $\phi \in C_{H}$ is such that the solution $x_{t}(\theta)$ of (2.1) with $x_{0}(\phi)=\phi$ is defined on $[0, \infty)$ and $\left\|x_{t}(\phi)\right\| \leq H_{1}<H$ for $t \in[0, \infty)$, then $\Omega(\phi)$ is a nonempty, compact, invariant set and

$$
\operatorname{dist}\left(x_{t}(\phi), \Omega(\phi)\right) \longrightarrow 0, \text { as } t \longrightarrow \infty
$$

Lemma 2.2 (See [13]). Let $V(\phi): C_{H} \longrightarrow \mathbb{R}$ be a continuous functional satisfying a local Lipschitz condition. $V(0)=0$ and such that

(i) $W_{1}(|\phi(0)|) \leq V(\phi) \leq W_{2}(\|\phi\|)$ where $W_{1}(r), W_{2}(r)$ are wedges.

(ii) $V_{(2.1)}^{\prime}(\phi) \leq 0$, for $\phi \leq C_{H}$.

The the zero solution of (2.1) is uniformly stable. If we define $Z=\{\phi \in$ $\left.C_{H}: V_{(2.1)}^{\prime}(\phi)=0\right\}$, then the zero solution of (2.1) is asymptotically stable, provided that the largest invariant set in $Z$ is $Q=\{0\}$.

The following will be our main stability result for (1.1). 
Theorem 2.1. Consider system (1.2) with

$$
p(t, x, y, x(t-r(t)), y(t-r(t)), z) \equiv 0, f(x), f^{\prime}(x), g(y), g^{\prime}(y), h(y)
$$

continuous in their respective arguments. Suppose further that

(i) for some $a>0, \epsilon_{0}>0, h(y) \geq a+\epsilon_{0}$ for all $y$;

(ii) for some $b>0, \frac{g(y)}{y} \geq b$ for all $y \neq 0$;

(iii) for some $c_{0}, \frac{f(x)}{x} \geq c_{0}$ for all $x \neq 0$;

(iv) for some $c>0, f^{\prime}(x) \leq c$ for all $x$, where $a b-c>0$;

(v) for some constants $L, M,\left|f^{\prime}(x)\right| \leq L,\left|g^{\prime}(y)\right| \leq M$, for all $x, y$.

Then the zero solution of (1.2) is asymptotically stable, provided that

$$
\gamma<\min \left\{\frac{2 \epsilon_{0}(1-\beta)}{(L+M)(1-\beta)+(1+a) M}, \frac{2(a b-c)(1-\beta)}{a[(L+M)(1-\beta)+(1+a) L]}\right\} .
$$

Proof. Using the equivalent system form (1.2), our main tool is the following Lyapunov functional $V\left(x_{t}, y_{t}, z_{t}\right)$ defined as

$$
\begin{aligned}
V\left(x_{t}, y_{t}, z_{t}\right)= & \int_{0}^{x} f(\xi) d \xi+\int_{0}^{y} v h(v) d v \\
& +a^{-1} \int_{0}^{y} g(u) d u+\frac{1}{2} a^{-1} z^{2}+y z+a^{-1} f(x) y \\
& +\lambda \int_{-r(t)}^{0} \int_{t+s}^{t} y^{2}(\theta) d \theta d s+\delta \int_{-r(t)}^{0} \int_{t+s}^{t} z^{2}(\theta) d \theta d s,
\end{aligned}
$$

where $\lambda$ and $\delta$ are positive constants which will be determined later.

The Lyapunov functional $V=V\left(x_{t}, y_{t}, z_{t}\right)$ defined in (2.2) can be arranged in the form

$$
\begin{aligned}
V\left(x_{t}, y_{t}, z_{t}\right)= & \frac{1}{2 a}(a y+z)^{2}+\frac{1}{2 a b}(f(x)+b y)^{2}+\int_{0}^{y}[h(v)-a] v d v \\
& +\frac{1}{a} \int_{0}^{y}\left[\frac{g(v)}{v}-b\right] v d v+\frac{1}{a b} \int_{0}^{x}\left[a b-f^{\prime}(s)\right] f(s) d s \\
& +\lambda \int_{-r(t)}^{0} \int_{t+s}^{t} y^{2}(\theta) d \theta d s+\delta \int_{-r(t)}^{0} \int_{t+s}^{t} z^{2}(\theta) d \theta d s .
\end{aligned}
$$


On using (i), (ii), (iii) and (iv) of Theorem (2.1), we obtain

$$
\begin{aligned}
V\left(x_{t}, y_{t}, z_{t}\right) \geq & \frac{1}{2 a}(a y+z)^{2}+\frac{1}{2 a b}(f(x)+b y)^{2} \\
& +\frac{1}{2} \epsilon_{0} y^{2}+\frac{c_{0}}{2 a b}(a b-c) x^{2}+\lambda \int_{-r(t)}^{0} \int_{t+s}^{t} y^{2}(\theta) d \theta d s \\
& +\delta \int_{-r(t)}^{0} \int_{t+s}^{t} z^{2}(\theta) d \theta d s .
\end{aligned}
$$

Since the integrals

$$
\lambda \int_{-r(t)}^{0} \int_{t+s}^{t} y^{2}(\theta) d \theta d s \text { and } \delta \int_{-r(t)}^{0} \int_{t+s}^{t} z^{2}(\theta) d \theta d s
$$

are non-negative,

$$
V\left(x_{t}, y_{t}, z_{t}\right) \geq \frac{1}{2 a}(a y+z)^{2}+\frac{1}{2 a b}(f(x)+b y)^{2}+\frac{1}{2} \epsilon_{0} y^{2}+\frac{c_{0}}{2 a b}(a b-c) x^{2} .
$$

Thus, we can find a positive constant $D_{1}$, small enough such that

$$
V\left(x_{t}, y_{t}, z_{t}\right) \geq D_{1}\left(x^{2}+y^{2}+z^{2}\right) .
$$

Next, our target is to show that $V\left(x_{t}, y_{t}, z_{t}\right)$ satisfies the conditions of Lemma 2.2. First, by (1.2) and (2.2), we obtain

$$
\begin{aligned}
\frac{d}{d t} V\left(x_{t}, y_{t}, z_{t}\right)= & -\frac{1}{a}(h(y)-a) z^{2}-\frac{1}{a}\left(a \frac{g(y)}{y}-f^{\prime}(x)\right) y^{2} \\
& +\left(y+\frac{1}{a} z\right)\left\{\int_{t-r(t)}^{t} g^{\prime}(y(s)) z(s) d s\right. \\
& \left.+\int_{t-r(t)}^{t} f^{\prime}(x(s)) y(s) d s\right\}+\lambda y^{2} r(t)+\delta z^{2} r(t) \\
& -\lambda\left(1-r^{\prime}(t)\right) \int_{t-r(t)}^{t} y^{2}(\theta) d \theta-\delta\left(1-r^{\prime}(t)\right) \\
& \times \int_{t-r(t)}^{t} z^{2}(\theta) d \theta
\end{aligned}
$$


By (v) and using $2 u v \leq u^{2}+v^{2}$, we obtain

$$
\begin{aligned}
\frac{d}{d t} V\left(x_{t}, y_{t}, z_{t}\right) \leq & -\frac{1}{a}(h(y)-a) z^{2}-\frac{1}{a}\left(a \frac{g(y)}{y}-f^{\prime}(x)\right) y^{2} \\
& +\frac{1}{2 a}(L+M+2 a \delta) z^{2} r(t)+\frac{1}{2}(L+M+2 a \lambda) y^{2} r(t) \\
& +\frac{L}{2 a}\left[1+a-2 \frac{a \lambda}{L}(1-\beta)\right] \int_{t-r(t)}^{t} y^{2}(s) d s \\
& +\frac{M}{2 a}\left[1+a-2 \frac{a \delta}{M}(1-\beta)\right] \int_{t-r(t)}^{t} z^{2}(s) d s,
\end{aligned}
$$

since $r^{\prime}(t) \leq \beta, 0<\beta<1$.

If we choose $\lambda=\frac{(1+a) L}{2 a(1-\beta)}>0$, and $\delta=\frac{(1+a) M}{2 a(1-\beta)}>0$, and using (i), (ii), (iv) and $r(t) \leq \gamma$, we obtain

$$
\begin{aligned}
\frac{d}{d t} V\left(x_{t}, y_{t}, z_{t}\right) \leq & -\frac{1}{2 a}\left\{2 \epsilon_{0}-\gamma\left(\frac{(L+M)(1-\beta)+(1+a) M}{(1-\beta)}\right)\right\} z^{2} \\
& -\frac{1}{2 a}\left\{2(a b-c)-\gamma\left(\frac{a[(L+M)(1-\beta)+(1+a) L]}{(1-\beta)}\right)\right\} y^{2},
\end{aligned}
$$

choosing

$$
\gamma<\min \left[\frac{2 \epsilon_{0}(1-\beta)}{(L+M)(1-\beta)+(1+a) M}, \frac{2(1-\beta)(a b-c)}{a[(L+M)(1-\beta)+(1+a) L]}\right],
$$

we have

$$
\frac{d}{d t} V\left(x_{t}, y_{t}, z_{t}\right) \leq-K\left(y^{2}+z^{2}\right) \text { for some } K>0
$$

Finally, it follows that $\frac{d}{d t} V\left(x_{t}, y_{t}, z_{t}\right) \equiv 0$ if and only if $y_{t}=z_{t}=0$, $\frac{d}{d t} V(\phi)<0$ for $\phi \neq 0$ and $V(\phi) \geq u(|\phi(0)|) \geq 0$. Thus, in view of (2.3), (2.4) and the last discussion, it is seen that all the conditions of Lemma 2.2 are satisfied. This shows that the trivial solution of Eq. (1.1) is asymptotically stable. Hence the proof of Theorem 2.1 is complete.

Remark 2.1. If $h\left(x^{\prime}\right)=a$ in (1.1), then Theorem 2.1 reduces to Theorem 1 of [13] and a result of [1]. 
Remark 2.2. If $h\left(x^{\prime}\right)=a, f(x(t-r(t)))=c x(t)$ in (1.1), then Theorem 2.1 reduces to Theorem 2 of [15].

Example 1.1. Consider the third order nonlinear delay differential equation

$$
\begin{gathered}
x^{\prime \prime \prime}(t)+\left[x^{\prime 2}(t)+x^{\prime}(t)+2\right] x^{\prime \prime}(t)+4 x^{\prime}(t-r(t)) \\
+\sin x^{\prime}(t-r(t))+\frac{x(t-r(t))}{1+x^{2}(t-r(t))}=0
\end{gathered}
$$

or its equivalent system form

$$
\begin{aligned}
x^{\prime}= & y, \\
y^{\prime}= & z, \\
z^{\prime}= & -\left[y^{2}+y+2\right] z(t)-[4 y+\sin y]-\frac{x}{1+x^{2}} \\
& +\int_{t-r(t)}^{t}(4+\cos y(s)) z(s) d s+\int_{t-r(t)}^{t} \frac{1-x^{2}(s)}{\left(1+x^{2}(s)\right)^{2}} y(s) d s
\end{aligned}
$$

where we suppose that $0 \leq r(t) \leq \gamma, r^{\prime}(t) \leq \beta, \beta$ and $\gamma$ are positive constants, $\gamma$ will be determined later, $t \in[0, \infty)$. It is obvious that

$$
3 \leq 4+\frac{\sin y}{y} \text { for all } y,(y \neq 0), \quad 1<y^{2}+y+2 \text { for all } y .
$$

Our main tool is the Lyapunov functional

$$
\begin{aligned}
V\left(x_{t}, y_{t}, z_{t}\right)= & \frac{1}{2}(y+z)^{2}+\frac{1}{6}\left(\frac{x}{1+x^{2}}+3 y\right)^{2} \\
& +\int_{0}^{y}\left[\left(v^{2}+v+2\right)-1\right] v d v+\int_{0}^{y}\left(1+\frac{\sin v}{v}\right) v d v \\
& +\frac{1}{3} \int_{0}^{x}\left(3-\frac{1-\xi}{\left(1+\xi^{2}\right)^{2}}\right) \frac{\xi}{1+\xi^{2}} d \xi \\
& +\lambda \int_{-r(t)}^{0} \int_{t+s}^{t} y^{2}(\theta) d \theta d s+\delta \int_{-r(t)}^{0} \int_{t+s}^{t} z^{2}(\theta) d \theta d s
\end{aligned}
$$

where $\lambda$ and $\delta$ are some positive constants which will be determined later. 
It is clear that the functional $V\left(x_{t}, y_{t}, z_{t}\right)$ is positive definite. Hence it is evident from the terms contained in (2.7), that there exist sufficiently small positive constant $\delta_{i},(i=1,2,3)$ such that

$$
\begin{gathered}
V\left(x_{t}, y_{t}, z_{t}\right) \geq \delta_{1} x^{2}+\delta_{2} y^{2}+\delta_{3} z^{2}+\lambda \int_{-r(t)}^{0} \int_{t+s}^{t} y^{2}(\theta) d \theta d s \\
+\delta \int_{-r(t)}^{0} \int_{t+s}^{t} z^{2}(\theta) d \theta d s \geq \delta_{1} x^{2}+\delta_{2} y^{2}+\delta_{3} z^{2} \geq \delta_{4}\left(x^{2}+y^{2}+z^{2}\right)
\end{gathered}
$$

where $\delta_{4}=\min \left\{\delta_{1}, \delta_{2}, \delta_{3}\right\}$.

Now, the time derivative of the functional $V\left(x_{t}, y_{t}, z_{t}\right)$ in (2.7) with respect to the system (2.6) can be calculated as follows:

$$
\begin{aligned}
\frac{d}{d t} V\left(x_{t}, y_{t}, z_{t}\right)= & -\left[\left(1+y+y^{2}\right)-\delta r(t)\right] z^{2} \\
& -\left[\left(4+\frac{\sin y}{y}\right)-\frac{1-x^{2}}{\left(1+x^{2}\right)^{2}}-\lambda r(t)\right] y^{2} \\
& +(y+z)\left[\int_{t-r(t)}^{t}(4+\cos y(s)) z(s) d s\right. \\
& \left.+\int_{t-r(t)}^{t} \frac{1-x^{2}(s)}{\left(1+x^{2}(s)\right)^{2}} y(s) d s\right]-\lambda\left(1-r^{\prime}(t)\right) \\
& \times \int_{t-r(t)}^{t} y^{2}(s) d s-\delta\left(1-r^{\prime}(t)\right) \int_{t-r(t)}^{t} z^{2}(\theta) d \theta .
\end{aligned}
$$

Making use of the fact that

$$
\begin{gathered}
|4+\cos y(s)| \leq 5,\left|\frac{\sin y}{y}\right| \leq 1,\left|\frac{1-x^{2}}{\left(1+x^{2}\right)^{2}}\right| \leq 1, \\
0 \leq r(t) \leq \gamma, r^{\prime}(t) \leq \beta, 0<\beta<1
\end{gathered}
$$

and the inequality $2|u v| \leq u^{2}+v^{2}$, we obtain the following inequalities for all terms contained in the inequality (2.8), respectively:

$$
\begin{aligned}
& -\left[\left(1+y+y^{2}\right)-\delta r(t)\right] z^{2} \leq-(1-\delta \gamma) z^{2} ; \\
& -\left[\left(4+\frac{\sin y}{y}\right)-\frac{1-x^{2}}{\left(1+x^{2}\right)^{2}}-\lambda r(t)\right] y^{2} \leq-(2-\lambda \gamma) y^{2} ;
\end{aligned}
$$




$$
\begin{aligned}
(y+z) & \left\{\int_{t-r(t)}^{t}(4+\cos y(s)) z(s)+\int_{t-r(t)}^{t} \frac{1-x^{2}(s)}{\left(1+x^{2}(s)\right)^{2}} y(s) d s\right\} \\
\leq & \left(\frac{5}{2}+\frac{1}{2}\right) r(t) y^{2}(t)+\left(\frac{5}{2}+\frac{1}{2}\right) r(t) z^{2}(t) \\
+ & \left(\frac{1}{2}+\frac{1}{2}\right) \int_{t-r(t)}^{t} y^{2}(s) d s+\left(\frac{5}{2}+\frac{5}{2}\right) \int_{t-r(t)}^{t} z^{2}(s) d s \\
\leq & 3 \gamma y^{2}(t)+3 \gamma z^{2}(t)+\int_{t-r(t)}^{t} y^{2}(s) d s+5 \int_{t-r(t)}^{t} z^{2}(s) d s
\end{aligned}
$$

and

$$
-\delta\left(1-r^{\prime}(t)\right) \int_{t-r(t)}^{t} z^{2}(s) d s \leq-\delta(1-\beta) \int_{t-r(t)}^{t} z^{2}(s) d s .
$$

Gathering all these inequalities into (2.8), we have

$$
\begin{aligned}
\frac{d}{d t} V\left(x_{t}, y_{t}, z_{t}\right) \leq & -(1-(\delta+3) \gamma) z^{2}-(2-(\lambda+3) \gamma) y^{2} \\
& -(\delta(1-\beta)-5) \int_{t-r(t)}^{t} z^{2}(s) d s \\
& -(\lambda(1-\beta)-1) \int_{t-r(t)}^{t} y^{2}(\theta) d \theta .
\end{aligned}
$$

Let us choose $\delta=\frac{5}{1-\beta}$ and $\lambda=\frac{1}{1-\beta}$. Then, it is easy to see that

$$
\frac{d}{d t} V\left(x_{t}, y_{t}, z_{t}\right) \leq-\left(1-\left(\frac{8-3 \beta}{1-\beta}\right) \gamma\right) z^{2}-\left(2-\left(\frac{4-3 \beta}{1-\beta}\right) \gamma\right) y^{2}
$$

Now, in view of (2.9), one can conclude for some positive constants $v$ and $\rho$ that

$$
\frac{d}{d t} V\left(x_{t}, y_{t}, z_{t}\right) \leq-v y^{2}-\rho z^{2}
$$

provided

$$
\gamma<\min \left\{\frac{1-\beta}{8-3 \beta}, \frac{2(1-\beta)}{4-3 \beta}\right\} .
$$

It is also easy to see that $\frac{d}{d t} V\left(x_{t}, y_{t}, z_{t}\right) \equiv 0$ if and only if $z_{t}=y_{t}=0$, $\frac{d}{d t} V(\phi)<0$ for $\phi \neq 0$ and $V(\phi) \geq u(|\phi(0)|) \geq 0$. Thus, all the conditions of Lemma 2.2 are satisfied. This shows that the trivial solution of (2.5) is globally asymptotically stable. 


\section{The boundedness of solutions}

Now, we shall state and prove our main result on boundedness of (1.1) with $p\left(t, x(t), x^{\prime}(t), x(t-r(t)), x^{\prime}(t-r(t)), x^{\prime \prime}(t)\right) \neq 0$.

Theorem 3.1 Let all the conditions of Theorem 2.1 be satisfied, in addition assume that there are positive constants $H$ and $H_{1}$ such that the following conditions are satisfied for every $x, y$ and $z$ in

$$
\Omega:=\left\{(x, y, z) \in \mathbb{R}^{3}:|x|<H_{1},|y|<H_{1},|z|<H_{1}, H_{1}<H\right\} .
$$

(i) $|p(t, x(t), y(t), x(t-r(t)), y(t-r(t)), z(t))| \leq q(t)$,

where $\max q(t)<\infty$ and $q \in L^{1}(0, \infty)$ the space of integrable Lebesgue functions.

Then, there exists a finite positive constant $K_{1}$ such that the solution $x(t)$ of (1.1) defined by the initial functions

$$
x(t)=\phi(t), \quad x^{\prime}(t)=\phi^{\prime}(t), \quad x^{\prime \prime}(t)=\phi^{\prime \prime}(t)
$$

satisfies the inequalities

$$
|x(t)| \leq K_{1}, \quad\left|x^{\prime}(t)\right| \leq K_{1}, \quad\left|x^{\prime \prime}(t)\right| \leq K_{1}
$$

for all $t \geq t_{0}$, where $\phi \in C^{2}\left(\left[t_{0}-r, t_{0}\right], \mathbb{R}\right)$, provided that

$$
\gamma<\min \left\{\frac{2 \epsilon_{0}(1-\beta)}{(L+M)(1-\beta)+(1+a) M}, \frac{2(a b-c)(1-\beta)}{a[(L+M)(1-\beta)+(1+a) L]}\right\} .
$$

Proof. As in Theorem 2.1, the proof of this theorem also depends on the scaler differentiable Lyapunov functional $V=V\left(x_{t}, y_{t}, z_{t}\right)$ defined in (2.2). Now, since $p(t, x(t), y(t), x(t-r(t)), y(t-r(t)), z(t)) \neq 0$, in view of (2.2), (1.2) and (2.4), it can be easily followed that the derivative of the functional $V\left(x_{t}, y_{t}, z_{t}\right)$ along (1.2) satisfies the following inequality,

$$
\begin{aligned}
\frac{d}{d t} V\left(x_{t}, y_{t}, z_{t}\right) & \leq-K\left(y^{2}+z^{2}\right)+\left|y+a^{-1} z\right| \\
& \times|p(t, x(t), y(t), x(t-r(t)), y(t-r(t)), z(t))| \\
& \leq-K\left(y^{2}+z^{2}\right)+\left|y+a^{-1} z\right| q(t) .
\end{aligned}
$$


Hence it follows that

$$
\begin{aligned}
\frac{d}{d t} V\left(x_{t}, y_{t}, z_{t}\right) & \leq-K\left(y^{2}+z^{2}\right)+D_{2}(|y|+|z|) q(t) \\
& \leq D_{2}(|y|+|z|) q(t)
\end{aligned}
$$

for a constant $D_{2}>0$, where $D_{2}=\max \left\{1, a^{-1}\right\}$.

Making use of the inequalities $|y|<1+y^{2}$ and $|z|<1+z^{2}$, it is clear that

$$
\frac{d}{d t} V\left(x_{t}, y_{t}, z_{t}\right) \leq D_{2}\left(2+y^{2}+z^{2}\right) q(t) .
$$

By (2.3), we have

$$
\left(x^{2}+y^{2}+z^{2}\right) \leq D_{1}^{-1} V\left(x_{t}, y_{t}, z_{t}\right)
$$

hence

$$
\frac{d}{d t} V\left(x_{t}, y_{t}, z_{t}\right) \leq D_{2}\left(2+D_{1}^{-1} V\left(x_{t}, y_{t}, z_{t}\right)\right) q(t) .
$$

Now, integrating the last inequality from 0 to $t$, using the assumption $q \in$ $L(0, \infty)$ and Gronwall-Reid-Bellman inequality, we obtain

$$
\begin{aligned}
V\left(x_{t}, y_{t}, z_{t}\right) & \leq V\left(x_{0}, y_{0}, z_{0}\right)+2 D_{2} A+D_{2} D_{1}^{-1} \int_{0}^{t}\left(V\left(x_{s}, y_{s}, z_{s}\right)\right) q(s) d s \\
& \leq\left(V\left(x_{0}, y_{0}, z_{0}\right)+2 D_{2} A\right) \exp \left(D_{2} D_{1}^{-1} \int_{0}^{t} q(s) d s\right) \\
& \leq\left(V\left(x_{0}, y_{0}, z_{0}\right)+2 D_{2} A\right) \exp \left(D_{2} D_{1}^{-1} A\right)=K_{2}<\infty,
\end{aligned}
$$

where $K_{2}>0$ is a constant, $K_{2}=\left(V\left(x_{0}, y_{0}, z_{0}\right)+2 D_{2} A\right) \exp \left(D_{2} D_{1}^{-1} A\right)$ and $A=\int_{0}^{\infty} q(s) d s$.

Now, the inequalities (2.3) and (3.1) together yield that

$$
x^{2}+y^{2}+z^{2} \leq D_{1}^{-1} V\left(x_{t}, y_{t}, z_{t}\right) \leq K_{3},
$$

where $K_{3}=K_{2} D_{1}^{-1}$. Thus, we conclude that

$$
|x(t)| \leq K_{3}, \quad|y(t)| \leq K_{3}, \quad|z(t)| \leq K_{3}
$$

for all $t \geq t_{0}$. That is

$$
|x| \leq K_{3}, \quad\left|x^{\prime}(t)\right| \leq K_{3}, \quad\left|x^{\prime \prime}(t)\right| \leq K_{3}
$$

for all $t \geq t_{0}$.

The proof of the theorem is now complete. 
Example 3.1. Consider the third order nonlinear delay differential equation

$$
\begin{gathered}
x^{\prime \prime \prime}(t)+\left[x^{\prime 2}(t)+x^{\prime}(t)+2\right] x^{\prime \prime}(t)+4 x^{\prime}(t-r(t)) \\
+\sin x^{\prime}(t-r(t))+\frac{x(t-r(t))}{1+x^{2}(t-r(t))} \\
=\frac{2}{1+t^{2}+x^{2}(t)+x^{\prime 2}(t)+x^{2}(t-r(t))+x^{\prime 2}(t-r(t))+x^{\prime \prime 2}(t)}
\end{gathered}
$$

or its equivalent system form

$$
\begin{aligned}
x^{\prime}= & y, \\
y^{\prime}= & z \\
z^{\prime}= & -\left[y^{2}+y+2\right] z-[4 y+\sin y]-\frac{x}{1+x^{2}} \\
& +\int_{t-r(t)}^{t}(4+\cos y(s)) z(s)+\int_{t-r(t)}^{t} \frac{1-x(s)}{\left(1+x^{2}(s)\right)^{2}} y(s) d s \\
& +\frac{2}{1+t^{2}+x^{2}+y^{2}+x^{2}(t-r(t))+y^{2}(t-r(t))+z^{2}(t)} .
\end{aligned}
$$

Observe that

$$
\frac{2}{1+t^{2}+x^{2}+y^{2}+x^{2}(t-r(t))+y^{2}(t-r(t))+z^{2}} \leq \frac{2}{1+t^{2}}=q(t)
$$

for all $t \in \mathbb{R}^{+}, x, y, x(t-r(t)), y(t-r(t)), z$ and

$$
\int_{0}^{\infty} q(s) d s=\int_{0}^{\infty} \frac{2}{1+s^{2}} d s=\pi<\infty, \text { that is } q \in L^{1}(0, \infty) .
$$

To show the boundedness of solutions we use as a main tool the Lyapunov functional (2.7). Now, in view of (2.10), the time derivative of the functional $V\left(x_{t}, y_{t}, z_{t}\right)$ with respect to the system (3.3) can be revised as follows:

$$
\begin{gathered}
\frac{d}{d t} V\left(x_{t}, y_{t}, z_{t}\right) \leq \\
-v y^{2}-\rho z^{2}+\frac{y+a^{-1} z}{1+t^{2}+x^{2}+y^{2}+x^{2}(t-r(t))+y^{2}(t-r(t))+z^{2}} .
\end{gathered}
$$


Making use of the fact

$$
\frac{1}{1+t^{2}+x^{2}+y^{2}+x^{2}(t-r(t))+y^{2}(t-r(t))+z^{2}} \leq \frac{1}{1+t^{2}}
$$

we get

$$
\frac{d}{d t} V\left(x_{t}, y_{t}, z_{t}\right) \leq-v y^{2}-\rho z^{2}+\frac{2\left|y+a^{-1} z\right|}{1+t^{2}} .
$$

Hence it is obvious that

$$
\begin{aligned}
\frac{d}{d t} V\left(x_{t}, y_{t}, z_{t}\right) & \leq \frac{2|y+z|}{1+t^{2}} \leq \frac{2|y|+|z|}{1+t^{2}} \\
& \leq \frac{2\left(2+y^{2}+z^{2}\right)}{1+t^{2}}=\frac{4}{1+t^{2}}+\frac{2\left(y^{2}+z^{2}\right)}{1+t^{2}} \\
& \leq \frac{4}{1+t^{2}}+\frac{2 D_{1}^{-1}}{1+t^{2}} V\left(x_{t}, y_{t}, z_{t}\right)
\end{aligned}
$$

Now, integrating (3.4) from 0 to $t$, using the fact $\frac{1}{1+t^{2}} \in L^{1}(0, \infty)$ and Gronwall-Reid-Bellman inequality, it can be easily concluded the boundedness of all solutions of (3.2).

\section{REFERENCES}

[1] A.U. Afuwape and M.O. Omeike, On the stability and boundedness of solutions of a kind of third order delay differential equations. Appl. Math. Comput., 200 (2008), 444-451.

[2] T.A. Burton, Volterra Integral and Differential Equations. Academic Press New York (1983).

[3] T.A. Burton, Stability and Periodic Solutions of Ordinary and Functional Differential Equations. Academic Press (1985).

[4] T.A. Burton and S. Zhang, Unified boundedness, periodicity, and stability in ordinary and functional differential equations. Ann Mat. Pure Appl., 145 (1986), 129-158.

[5] L.É. Él'sgol'ts, Introduction to the theory of differential equations with deviating arguments. Translated from the Russian by Robert J. McLaughlin Holden-Day, Inc., San Francisco, Calif.-London-Amsterdam (1966).

[6] K. Gopalsamy, Stability and Oscillations in Delay Differential Equations of Population Dynamics. Kluwer Academic, Dordrecht (1992).

[7] J.K. Hale, Theory of Functional Differential Equations. Springer-Verlag, New York (1977).

[8] V. Kelmanovskii and A. Myshkis, Introduction to the Theory and Applications of Functional Differential Equations. Kluwer Academic, Dordrecht (1999). 
[9] S. Li and L. Wen, Functional Differential Equations. Human Science and Technology Press (1987).

[10] G. Makay, On the asymptotic stability of the solutions of functional differential equations with infinite delay. J. Diff. Eqs., 108 (1994), 139-151.

[11] Y. Qin, Y. Liu and L. Wang, Stability of Motion for Dynamics Systems with Delay. Academic Press, Beijing (1966).

[12] R. Reissig, E. Sansone and R. Conti, Non-linear Differential Equations of Higher Order. Noordhoff, Groningen (1974).

[13] A.I. Sadek, Stability and Boundedness of a Kind of Third Order Delay Differential System. Applied Mathematics Letters, 16 (2003), 657-662.

[14] H.O. Tejumola, A note on the Boundedness and the Stability of Solutions of Certain Third Order Differential Equations. Ann. Mat. Pura. Appl. (iv), 92 (1972), 65-75.

[15] C. Tunç, Stability and boundedness of solutions of nonlinear equations of third-order with delay. Differential Equations and Control Processes, 3 (2007), 1-13.

[16] C. Tunç, On asymptotic stability of solutions to third order nonlinear differential equations with retarded argument. Commun. Appl. Anal., 11(3-4) (2007), 515-527.

[17] C. Tunç, On the boundedness of solutions of third-order differential equations with delay. (Russian) Differ. Uravn., 44(4) (2008), 446-454, 574.

[18] C. Tunç, A new boundedness result to nonlinear differential equations of third order with finite lag. Commun. Appl. Anal., 13(1) (2009), 1-10.

[19] C. Tunç, Stability criteria for certain third order nonlinear delay differential equations. Portugaliae Mathematica, 66(1) (2009), 71-80.

[20] T. Yoshizawa, Stability Theorem by Liapunov's Second Method. The Mathematical Society of Japan (1966).

[21] Y. Zhu, On Stability, Boundedness and existence of periodic solution of a kind of thirdorder nonlinear delay differential system. Ann. Diff. Eqs., 8(2) (1992), 249-259. 